\title{
Thermopower of single-channel disordered and chaotic conductors
}

\author{
S A van Langen, P G Silvestrovt, C W J Bennakker \\ Instituut-Lorentz, Leiden Unversity, PO Box 95062300 RA Leiden The Netherlands
}

(Recelved 30 October 1997)

\begin{abstract}
We show (analytically and by numerical simulation) that the zero-temperature limit of the distribution of the theimopower $S$ of a one-dimensional disordeied wire in the localized regime is a Lorentzian, with a disolder-1ndependent width of $4 \pi^{3} k_{\mathrm{B}}^{2} T / 3 e \Delta$ (where $T$ is the temperature and $\Delta$ the mean level spacing) Upon iassing the temperature the distribution crosses over to an exponentral form $\propto \exp (-2|S| e T / \Delta)$ We also consider the case of a chaotic quantum dot with two single channel ballistic point contacts The distribution of $S$ then has a cusp at $S=0$ and a tail $\propto|S|^{1}{ }^{\beta} \ln |S|$ for large $S$ (w1th $\beta=1,2$ depending on the presence or absence of time reversal symmetry)

(C) 1998 Academıc Piess Limıted
\end{abstract}

Key words: thermo electı1c phenomena, localızation, quantum chaos

\section{Introduction}

Thermo-electric transpoit proper ties of conductors probe the energy dependence of the scattering processes limiting conduction At low temperatues and in small (mesoscopic) systems, elastic impurity scattering is the dominant scattering process The energy dependence of the conductance is then a quantum interference effect [1] The derivative $d G / d E$ of the conductance with iespect to the Fermi eneigy is measured by the theimopower $S$, defined as the 1 atı $-\Delta V / \Delta T$ of a (small) voltage and temperature difference applied over the sample at zero electıc current Experımental and theoretıcal studies of the thermopower exist for several mesoscopic devices One finds a selles of sharp peaks in the thermopower of quantum point contacts [2], aperiodic fluctuations in diffusive conductors [3], sawtooth oscillations in quantum dots in the Coulomb blockade 1egime [4], and Aharonov-Bohm oscillations in metal ıngs [5]

Heie we study the statistical distribution of the thermopower in two different systems, not consideied previously A disordeied wire in the localized 1egime and a chaotic quantum dot with ballistic point contacts A single transmitted mode is assumed in both cases In the disordered wire, conduction takes place by resonant tunnelling through localized states The resonances ate very narrow and appear at uncorrelated energies The distributions of the thermopower and the conductance are both bioad, but otherwise quite different instead of the log normal distiıbution of the conductance [1] we find a Lorentzian distisbution for the thermopowes In the quantum dot, the resonances ate cortelated and the widths ate of the same order as the spacings The coirelations are descised by 1 andom-matrix theory $[6,7]$, under the assumption that the classical dynamics in the dot is chaotic The theimopower disti1bution in this case follows from the distribution of the time delay matrix found recently [8]

I Also at Budkeı Institute of Nuclear Physics Novosıb11sk, Russia 
The thermopower (at temperature $T$ and Fermi energy $E_{\mathrm{F}}$ ) is given by the Cutler-Mott formula $[9,10]$

$$
S=-\frac{1}{e T} \frac{\int d E\left(E-E_{\mathrm{F}}\right) G(E) d f / d E}{\int d E G(E) d f / d E},
$$

where $G$ is the zero-temperature conductance and $f$ is the Fermi-Dirac distribution function. In the limit $T \rightarrow 0$ eqn (1) simplifies to

$$
S=-\frac{\pi^{2}}{3} \frac{k_{\mathrm{B}}^{2} T}{e G} \frac{d G}{d E}
$$

where $G$ and $d G / d E$ are to be evaluated at $E=E_{\mathrm{F}}$. We consider mainly the zero-temperature limit of the thermopower, by studying the dimensionless quantity

$$
\sigma=\frac{\Delta}{2 \pi G} \frac{d G}{d E}
$$

Here $\Delta$ is the mean level spacing near the Fermi energy. Since we are dealing with single-channel conduction, the conductance is related to the transmission probability $T(E)$ by the Landauer formula [1, 11]

$$
G(E)=\frac{2 e^{2}}{h} T(E)
$$

The problem of the distribution of the thermopower is therefore a problem of the distribution of the logarithmic derivative of the transmission probability.

\section{Disordered wire}

In this section we study a disordered single-mode wire of length $L$ much greater than the mean free path $l$. This is the localized regime. We compute the thermopower distribution in the zero-temperature limit. The analytical theory is tested by comparing with a numerical simulation. The effect of a finite temperature is considered at the end of the section. Electron-electron interactions play an important role in one-dimensional conduction, but we do not take these into account here.

\subsection{Analytical theory}

The localization length $\xi(E)$ (which is of order $l$ and is defined by $\lim _{L \rightarrow \infty} L^{-1} \operatorname{In} T(E)=-2 / \xi(E)$ ) and the density of states $\rho(E)$ (per unit of length in the limit $L \rightarrow \infty$ ) are related by the Herbert-Jones-Thouless formula [12]

$$
\frac{1}{\xi(E)}=\int d E^{\prime} \rho\left(E^{\prime}\right) \ln \left|E-E^{\prime}\right|+\text { constant. }
$$

The additive constant is energy independent on the scale of the level spacing. Equation (5) follows from the Kramers-Kronig relation between the real and imaginary parts of the wavenumber (the real part determining $\rho$, the imaginary part $\xi$ ). Neglecting the width of the resonances in the large- $L$ limit, the density of states $\rho(E)=L^{-1} \sum_{i} \delta\left(E-E_{i}\right)$ is a sum of delta functions, and thus

$$
\sigma=-\frac{L \Delta}{\pi} \frac{d}{d E} \frac{1}{\xi(E)}=\frac{\Delta}{\pi} \sum_{i} \frac{1}{E_{i}-E_{\mathrm{F}}} .
$$

In the localized regime the energy levels $E_{i}$ are uncorrelated, and we assume that they are uniformly distributed in a band of width $B$ around $E_{\mathrm{F}}$. To obtain the distribution of $\sigma$,

$$
P(\sigma)=\prod_{i} \int_{-B / 2}^{B / 2} \frac{d E_{i}}{B} \delta\left(\sigma-\frac{\Delta}{\pi} \sum_{j} \frac{1}{E_{j}}\right),
$$


we first compute the Fourier transform

$$
P(k)=\int_{-\infty}^{\infty} d \sigma \mathrm{e}^{\mathrm{ik \sigma}} P(\sigma)=\left[\frac{1}{B} \int_{-B / 2}^{B / 2} d E \mathrm{e}^{i k \Delta / \pi E}\right]^{B / \Delta}=\mathrm{e}^{-|k|}
$$

where the limit $B / \Delta \rightarrow \infty$ is taken in the last step. Inverting the Fourier transform, we find that the thermopower distribution is a Lorentzian,

$$
P(\sigma)=\frac{1 / \pi}{1+\sigma^{2}}
$$

The 'full width at half maximum' of $P(\sigma)$ is equal to 2 , hence it is equal to $4 \pi^{3} k_{\mathrm{B}}^{2} T / 3 e \Delta$ for $P(S)$. This width depends on the length $L$ of the system (through $\Delta \propto 1 / L$ ), but it does not depend on the mean free path $l$ (as long as $l \ll L$, so that the system remains in the localized regime).

\subsection{Numerical simulation}

In order to check the analytical theory, we performed a numerical simulation using the tight-binding Hamiltonian

$$
\mathcal{H}=-\frac{w}{2} \sum_{J}\left(c_{J+1}^{\dagger} c_{j}+c_{j}^{\dagger} c_{J+1}\right)+\sum_{j} V_{j} c_{j}^{\dagger} c_{j} .
$$

The disordered wire was modelled by a chain of lattice constant $a$, with a random impurity potential $V_{J}$ at each site drawn from a Gaussian distribution of mean zero and variance $u^{2}$. The localization length of the wire is given by $\xi=2\left(a / u^{2}\right)\left(w^{2}-E_{\mathrm{F}}^{2}\right)$ [13]. We have chosen $u=0.075 w, E_{\mathrm{F}}=-0.55 w$, such that $\xi=248 a$, much smaller than $L=8000$ a. From the scattering matrix we obtained the conductance via the Landauer formula (4), and then the (dimensionless) thermopower via eqn (3) (with $\Delta=3.3 \times 10^{-4} \mathrm{w}$ ). The differentiation with respect to energy was carried out numerically, by repeating the calculation at two closely spaced values of $E_{\mathrm{F}}$. As shown in Fig. 1, the agreement with the analytical result is good without any adjustable parameters.

\subsection{Finite temperatures}

Our derivation of the Lorentzian distribution of the thermopower holds if the temperature is so low that $k_{\mathrm{B}} T$ is small compared to the typical width $\gamma$ of the transmission resonances. What if $k_{\mathrm{B}} T>\gamma$, but still $k_{\mathrm{B}} T \ll \Delta$ (so that the discreteness of the spectrum remains resolved)? We will show that the distribution crosses over to an exponential, but in a highly nonuniform way.

Consider arbitrary $\gamma$ and $k_{\mathrm{B}} T$, both $\ll \Delta$. The Cutler-Mott formula (1) is dominated by two contributions, one from a peak in $d f / d E$ of width $k_{\mathrm{B}} T$ around $E_{\mathrm{F}}$ and one from a peak in $G(E)$ of width $\gamma_{0}$ around $E_{0}$. Here $\gamma_{0}$ and $E_{0}$ are the width and position of the level closest to $E_{\mathrm{F}}$. If $\left|E_{\mathrm{F}}-E_{0}\right| \gg \max \left(k_{\mathrm{B}} T, \gamma_{0}\right)$, the two peaks do not overlap and one can estimate the thermopower as

$$
S=\frac{1}{e T}\left[\frac{\pi \gamma_{0}\left(k_{\mathrm{B}} T\right)^{2}}{3\left(E_{\mathrm{F}}-E_{0}\right)^{3}}+\frac{E_{\mathrm{F}}-E_{0}}{k_{\mathrm{B}} T} \mathrm{e}^{-\left|E_{\mathrm{\imath}}-E_{0}\right| / k_{\mathrm{B}} T}\right]\left[\frac{\gamma_{0}}{2 \pi\left(E_{\mathrm{F}}-E_{0}\right)^{2}}+\frac{1}{k_{\mathrm{B}} T} \mathrm{e}^{-\left|E_{\mathrm{F}}-E_{0}\right| / k_{\mathrm{B}} T}\right]^{-1} .
$$

If $k_{\mathrm{B}} T \ll \gamma_{0}$, the first terms in the numerator and denominator dominate over the second terms. This is the regime that the Lorentzian distribution (9) holds for all $S$.

We now turn to the regime $k_{\mathrm{B}} T>\gamma_{0}$. The first terms dominate if $\left|E_{\mathrm{F}}-E_{0}\right| \gg k_{\mathrm{B}} T \ln k_{\mathrm{B}} T / \gamma_{0}$. Hence $P(S)$ is a Lorentzian for $|S| \ll\left(k_{\mathrm{B}} / e\right)\left(\ln k_{\mathrm{B}} T / \gamma_{0}\right)^{-1}$. The logarithm of $k_{\mathrm{B}} T / \gamma_{0}$ can be quite large, because the width of the levels is exponentially small in the system size, $\gamma \sim \mathrm{e}^{-L / \xi}$. The Lorentzian persists in an 


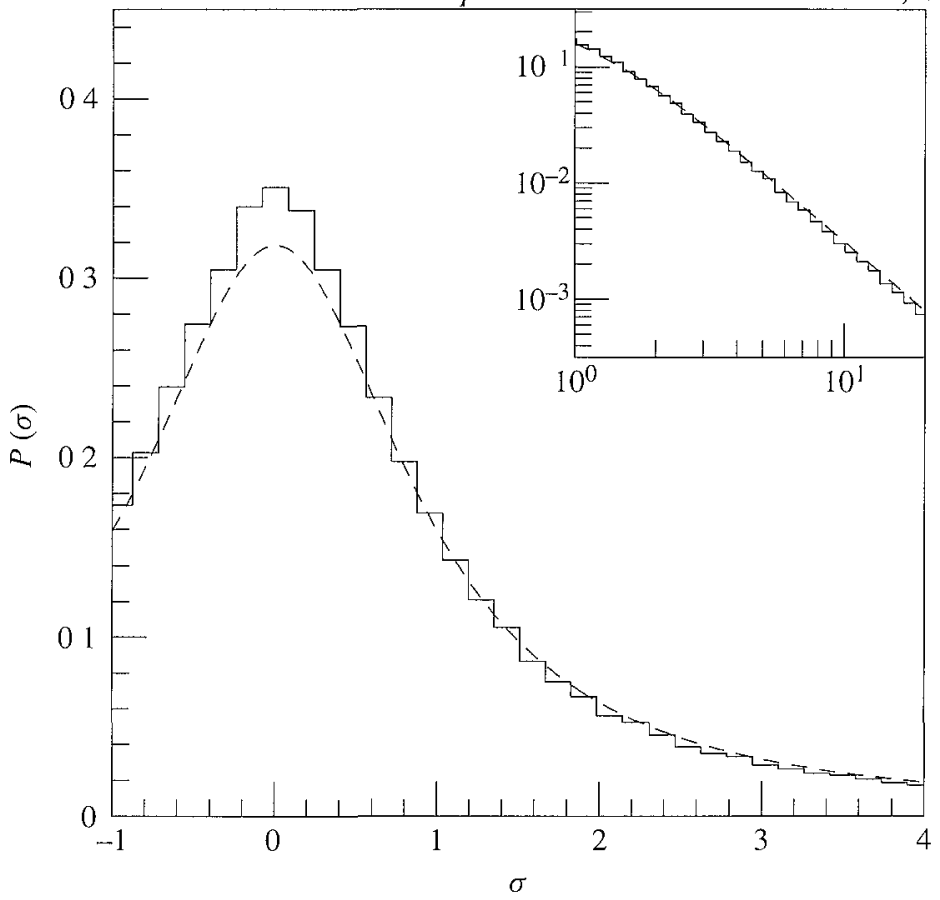

Fig. 1. Distribution of the dimensionless thei mopower $\sigma=(\Delta / 2 \pi) d \ln T(E) / d E$ for a one dimcnsional wive in the localized tegime The histogram is obtaned fiom a numerical simulation for a sample length $L=323 \xi$ The dashed cutve is the Lorentzian (9) being the analytical iesuit for $L \gg \xi$ The inset shows the algebraic tal of the distribution on a logatithmic scale The theimopowei $S$ in the zero temperature limit is ielated to $\sigma$ by $S=-\left(2 \pi^{3} / 3\right)\left(k_{\mathrm{B}}^{2} T / e \Delta\right) \sigma$

interval larger than its width, provided $k_{\mathrm{B}} T<\Delta\left(\ln k_{\mathrm{B}} T / \gamma_{0}\right)^{-1}$ The second terms in eqn (11) dominate if $k_{\mathrm{B}} T \ll\left|E_{\mathrm{F}}-E_{0}\right| \ll k_{\mathrm{B}} T \ln k_{\mathrm{B}} T / \gamma_{0}$ In this case the thermopower is simply $S=\left(E_{\mathrm{F}}-E_{0}\right) / e T$, with exponentral distribution

$$
P(S)=\frac{e T}{\Delta} \mathrm{e}^{2|S| e T / \Delta}
$$

The distribution (12) follows because the energy levels are uncorrelated, so that the spacing $\left|E_{\mathrm{F}}-E_{0}\right|$ has an exponential distribution with a mean of $\Delta / 2$

We conclude that the thermopower disti ibution for $\gamma<k_{\mathrm{B}} T \ll \Delta$ contains both Lorentzian and exponential contributions The peak region $|S| \ll\left(k_{\mathrm{B}} / e\right)\left(\ln k_{\mathrm{B}} T / \gamma\right)^{-1}$ is the Lorentzian (9) The intermediate iegion $\left(k_{\mathrm{B}} / e\right)\left(\ln k_{\mathrm{B}} T / \gamma\right)^{-1} \ll|S| \ll\left(k_{\mathrm{B}} / e\right) \ln k_{\mathrm{B}} T / \gamma$ is the exponentral (12) The far ta1ls $|S| \gg\left(k_{\mathrm{B}} / e\right) \ln k_{\mathrm{B}} T / \gamma$ cannot be explained by eqn (11) With increasing temperature, the Lorentzian peak region shinks, and ultimately the exponential region starts 1 ght at $S=0$ This applies to the temperature 1 ange $\Delta\left(\ln k_{\mathrm{B}} T / \gamma\right)^{-1}<$ $k_{\mathrm{B}} T \ll \Delta$

To illustrate these various regimes, we computed $P(S)$ numerically from eqn (1) We took the density of states

$$
\rho(E)=L^{-1} \sum_{l} \frac{\gamma_{l} / 2 \pi}{\left(E-E_{l}\right)^{2}+\gamma_{l}^{2} / 4},
$$

so that the conductance according to eqn (5) has the energy dependence

$$
G(E) \propto \prod_{l}\left[\left(E-E_{l}\right)^{2}+\gamma_{l}^{2} / 4\right]^{-1}
$$




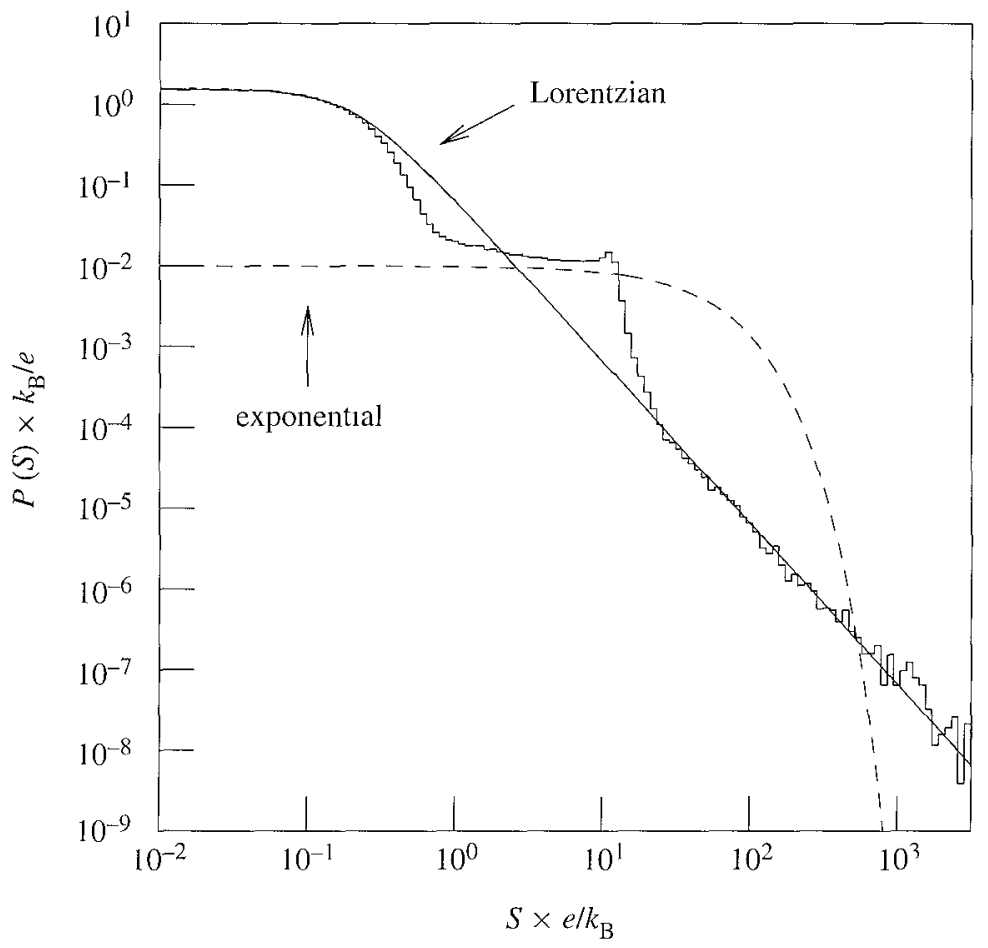

Fig. 2. Thermopowei distıbution of a one-dimensional wire in the localized iegime at finite tempeiature The histogiam 15 obtained from eqns (1) and (14), by numentcal integtation for a set of a andomly chosen energy levels $E_{t}$, all having the same width $\gamma_{1}=\gamma=10^{-6} \Delta$ The temperature is $k_{\mathrm{B}} T / \Delta=00 \mathrm{I}$, such that $\gamma \ll k_{\mathrm{B}} T \ll \Delta$ The distibution follows the Lorentzian (9) (solid curve) for small and laige $S$, but it follows the exponential (12) (dashed curve) in an mteimediate region

The levels $E_{i}$ were chosen uniformly and independently (mean spacing $\Delta$ ), but the fluctuations of the widths $\gamma_{1}$ were ignored $\left(\gamma_{\imath} \equiv \gamma\right.$ for all $i$ ). Such fluctuations are irrelevant in the low-temperature limit $k_{\mathrm{B}} T \ll \gamma$, but not for $\gamma<k_{\mathrm{B}} T \ll \Delta$. We believe that ignoring fluctuations in $\gamma_{r}$ should still be a reasonable approximation, because $\gamma_{0}$ appears only in logarithms. The resulting $P(S)$ is plotted in Fig. 2 . We see the expected crossover from a Lorentzian to an exponential. The exponential region appears as a plateau. Beyond the exponential region, the distribution appears to return to the Lorentzian form. We have no explanation for this far tail.

\section{Chaotic quantum dot}

In this section we consider a chaotic quantum dot with single-channel ballistic point contacts (see Fig. 3, inset). Because there are no tunnel barriers in the point contacts, the effects of the Coulomb blockade are small and here we ignore them altogether. For this system, the distribution of $d T / d E$ was computed recently from random-matrix theory [8]. The energy derivative of the transmission probability has the parametrization

$$
\frac{d T}{d E}=\frac{c}{\hbar}\left(\tau_{1}-\tau_{2}\right) \sqrt{T(1-T)}
$$

with independent distributions

$$
\begin{aligned}
P(c) & \propto\left(1-c^{2}\right)^{-1+\beta / 2}, \quad|c|<1, \\
P\left(\tau_{1}, \tau_{2}\right) & \propto\left|\tau_{1}-\tau_{2}\right|^{\beta}\left(\tau_{1} \tau_{2}\right)^{-2(\beta+1)} \mathrm{e}^{-\left(1 / \tau_{1}+1 / \tau_{2}\right) \pi \beta \hbar / \Delta}, \quad \tau_{1}, \tau_{2}>0,
\end{aligned}
$$




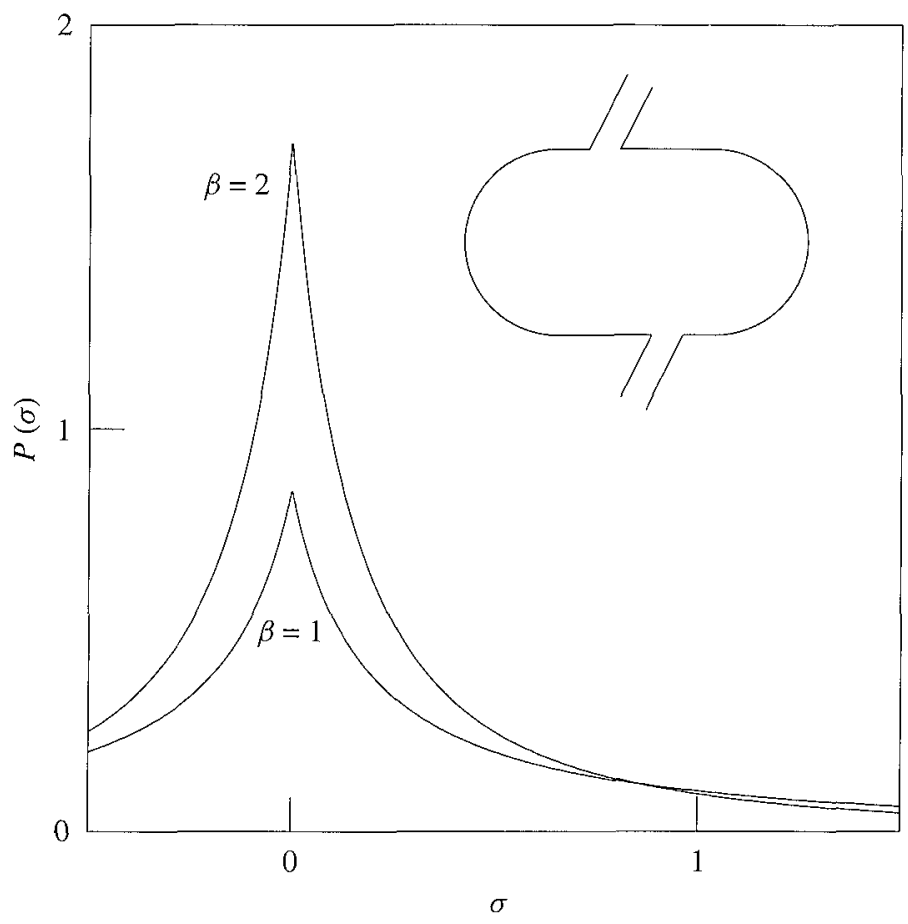

Fig. 3. Distribution of the dimensionless thermopower of a chaotic cavity with two single-channel ballistic point contacts (1nset), computed from eqn (19) for the case of broken $(\beta=2)$ and unbroken $(\beta=1)$ time-reversal symmetry

$$
P(T) \propto T^{-1+\beta / 2}, \quad 0<T<1 .
$$

The integer $\beta$ equals 1 or 2 , depending on whether time-reversal symmetry is present or not. The times $\tau_{1}, \tau_{2}$ are the eigenvalues of the Wigner-Smith time-delay matrix (see $[8,14]$ ). Their sum $\tau_{1}+\tau_{2}$ is the density of states (multiplied by $2 \pi \hbar$ ). The thermopower distribution follows from

$$
\begin{aligned}
P(\sigma) \propto & \int_{-1}^{1} d c P(c) \int_{0}^{\infty} d \tau_{1} \int_{0}^{\infty} d \tau_{2} P\left(\tau_{1}, \tau_{2}\right) \int_{0}^{1} d T P(T) \\
& \times\left(\tau_{1}+\tau_{2}\right) \delta\left(\sigma-(\Delta / 2 \pi \hbar) c\left(\tau_{1}-\tau_{2}\right) \sqrt{1 / T-1}\right)
\end{aligned}
$$

As in $[8,15]$, the density of states appears as a weight factor $\tau_{1}+\tau_{2}$ in the ensemble average (19), because the ensemble is generated by uniformly varying the charge on the quantum dot rather than its Fermi energy. This is the correct thing to do in the Hartree (self-consistent potential) approximation. A more sophisticated treatment of the electron-electron interactions (as advocated in [16]) does not yet exist for this problem. The resulting distributions are plotted in Fig. 3. The curves have a cusp at $\sigma=0$, and asymptotes $P(\sigma) \propto|\sigma|^{-1-\beta} \ln |\sigma|$ for $|\sigma| \gg 1$.

\section{Conclusion}

The results we have reported hold for single-channel conductors. The generalization to multi-channel conductors is of interest. Multi-channel diffusive conductors were studied in [3]. For a chaotic cavity with ballistic point contacts having a large number of modes ( $N$ modes per point contact), the distribution of the 
thermopower is Gaussian The mean is zero and the variance is

$$
\text { Vai } S=\frac{k_{\mathrm{B}}^{4} T^{2} \pi^{6}}{9 e^{2} N^{4} \Delta^{2} \beta}
$$

(We have used the results of [17] ) Analogously to universal conductance fluctuations, the variance of the thermopowe1 is 1educed by a factor of 2 upon breaking time-revessal symmetry $(\beta=1 \rightarrow \beta=2)$

For an $N$-mode wire in the localized tegime, our derivation of the exponential distribution of the thermopower iemains valıd This is not true for the Lorentzian distribution The reason is that the Herbert-JonesThouless for mula for $N>1$ telates the density of states to the sum of the inverse localization lengths, [18] and there is no simple relation between this sum and the thermopower We expect that the tail of the distribution remains quadiatıc, $P(S) \propto S^{-2}$-because of the argument of Section 23 , which is still valid for $N>1$ It re mains a challenge to determine analytically the entise thei mopower distribution of a multi channel disoidered w11e

Acknowledgements-This papei is dedicated to Rolf Landauer on the occasion of his 70th birthday Discussions with P W Brouwer are gratefully acknowledged This iesearch was suppoited by the 'Nedeilandse o1ganısatıe voor Wetenschappelıjk Onderzoek' (NWO) and by the 'St1chtıng voor Fundamenteel Ondeızoek der Materie' (FOM)

\section{References}

[1] Y Imry, Introduction to Mesoscopuc Physics, (Oxford University, Oxford, 1997)

[2] P Středa, J Phys C1, 1025 (1989), L W Molenkamp, Th Giavier, H van Houten, O J A Bu1jk, M A A Mabesoone, and C T Foxon, Phys Rev Lett 65, 1052 (1990), C R Proetto, Phys Rev B44, 9096 (1991), R A Wyss, C C Eugster, J A del Alamo, Q Hu, M J Rooks, and M R Melloch, Appl Phys Lett 66, 1144 (1995)

[3] A V Anısovich, B L Alt'shuler, A G Atonov, and A Yu Zyuzin, Pis'ma Zh Eksp Teor Fiz 45, 237 (1987) [JETP Lett 45, 295 (1987)], G B Lesovik and D E Khmel'nitskǐ̌, Zh Eksp Teor F1z 94, 164 (1988) [Sov Phys JETP 67, 957 (1988)], R A Selota, M Ma, and B Goodman, Phys Rev B37, 6540 (1988), G M Gusev, Z D Kvon, and A G Pogosov, P1s'ma Zh Eksp Teor Fiz 51, 151 (1990) [JETP Lett 51, 171 (1990)], B L Gallagher, T Galloway, P Beton, J P Oxley, S P Beaumont, S Thoms, and C D W Wilkinson, Phys Rev Lett 64, 2058 (1990), D P DiVincenzo, Phys Rev B48, 1404 (1993)

[4] C W J Beenakkeı and A A M Starıng, Phys Rev B46, 9667 (1992), A S Dzurak, C G Smith, M Pepper, D A Ritchie, J E F Frost, G A C Jones, and D G Hasko, Sol State Comm 87, 1145 (1993), A A M Staring, L W Molenkamp, B W Alphenaar, H van Houten, O J A Buık, M A A Mabesoone, C W J Beenakker, and C T Foxon, Euiophys Lett 22, 57 (1993), A S Dzurak, C G Smith, C H W Baınes, M Pepper, L Martın-Moieno, C T Liang, D A Ritchie, and G A C Jones, Phys Rev B55, R10197 (1997)

[5] Ya M Blanter, C Bruder, R Fazı, and H Schoeller, Phys Rev B55, 4069 (1997)

[6] C W J Beenakker, Rev Mod Phys 69,731 (1997)

[7] T Guhı, A Muller-Groelıng, and H A We1denmuller, Phys Rep (to be published)

[8] P W Brouwer, S A van Langen, K M Fiahm, M Buttıke, and C W J Beenakker, Phys Rev Lett 79, 913 (1997)

[9] M Cutle1 and N F Mott, Phys Rev 181, 1336 (1969)

[10] U Sivan and Y Imry, Phys Rev B33, 551 (1986)

[11] R Landauer, IBM J Res Dev 1, 223 (1957)

[12] D C Herbert and R Jones, J Phys C4, 1145 (1971), D J Thouless, J Phys C 5, 77 (1972)

[13] O N Doıokhov, Zh Eksp Teoı Fiz 101, 966 (1992) [Sov Phys JETP 74, 518 (1992)] 
[14] Y. V. Fyodorov and H.-J. Sommers, J. Math. Phys. 38, 1918 (1997).

[15] M. H. Pedersen, S. A. van Langen, and M. Büttiker, Phys. Rev. B (to be published).

[16] I. L. Aleiner and L. I. Glazman, preprints (cond-mat/9612138, 9710195).

[17] K. B. Efetov, Phys. Rev. Lett. 74, 2299 (1995); K. Frahm, Europhys. Lett. 30, 457 (1995).

[18] W. Craig and B. Simon, Comm. Math. Phys. 90, 207 (1983). 